\section{(C) OPEN ACCESS}

\begin{abstract}
- Additional material is published online only. To view, please visit the journal online (http://dx.doi.org/10.1136/ oemed-2017-104529).
\end{abstract}

For numbered affiliations see end of article.

Correspondence to Dr Xiang Qian Lao, Jockey Club School of Public Health and Primary Care, The Chinese University of Hong Kong, School of Public Health, Prince of Wales Hospital, Sha Tin, N.T, Hong Kong SAR, China;

xqlao@cuhk.edu.hk

Received 14 May 2017 Revised 13 September 2017 Accepted 11 October 2017

Published Online First

13 November 2017

\title{
Exposure to ambient fine particulate matter and semen quality in Taiwan
}

\author{
Xiang Qian Lao, ${ }^{1,2}$ Zilong Zhang, ${ }^{1}$ Alexis K H Lau, ${ }^{3,4}$ Ta-Chien Chan, ${ }^{5}$ \\ Yuan Chieh Chuang, ${ }^{6}$ Jimmy Chan, ${ }^{3}$ Changqing Lin, ${ }^{4}$ Cui Guo, ${ }^{1}$ Wun Kai Jiang, ${ }^{6}$ \\ Tony Tam, ${ }^{7}$ Gerard Hoek ${ }^{8}$ Haidong Kan, ${ }^{9}$ Eng-kiong Yeoh, ${ }^{1}$ Ly-yun Chang ${ }^{6,10}$
}

\section{ABSTRACT \\ Objectives Environmental exposure to chemicals} has been considered a potential factor contributing to deteriorated semen quality. However, previous literature on exposure to air pollution and semen quality is inconsistent. We therefore investigated the health effects of short-term and long-term exposure to fine particulate matter $\left(\mathrm{PM}_{2.5}\right)$ on semen quality in Taiwanese men from the general population.

Methods A cross-sectional study was conducted among 6475 male participants aged $15-49$ years who participated in a standard medical examination programme in Taiwan between 2001 and 2014. Semen quality was assessed according to the WHO 1999 guidelines, including sperm concentration, total motility, progressive motility and morphology. Three-month and 2-year average $\mathrm{PM}_{2.5}$ concentrations were estimated at each participant's address using a spatiotemporal model based on satellite-derived aerosol optical depth data. Multivariable linear and logistic regressions were used to examine the associations between $\mathrm{PM}_{2.5}$ and semen quality.

Results A robust association was observed between exposure to $\mathrm{PM}_{2.5}$ and decreased normal morphology. Every increment of $5 \mu \mathrm{g} / \mathrm{m}^{3}$ in 2-year average $\mathrm{PM}_{2.5}$ was significantly associated with a decrease of $1.29 \%$ in sperm normal morphology and a $26 \%$ increased risk of having the bottom $10 \%$ of sperm normal morphology, after adjusting for a wide range of potential confounders $(p<0.001)$. On the other hand, an increment of $5 \mu \mathrm{g} /$ $\mathrm{m}^{3}$ in 2-year average $\mathrm{PM}_{2.5}$ was associated with an increase of $1.03 \times 10^{6} / \mathrm{mL}$ in sperm concentration and a $10 \%$ decreased risk of being the bottom $10 \%$ of sperm concentration (both $p<0.001$ ). Similar results were found for 3-month $\mathrm{PM}_{2.5}$.

Conclusions Exposure to ambient $\mathrm{PM}_{2.5}$ air pollution is associated with a lower level of sperm normal morphology and a higher level of sperm concentration.

\section{INTRODUCTION}

Infertility is a global public health concern. It was estimated that 48.5 million couples worldwide were infertile in 2010, ${ }^{1}$ with men contributing $>50 \% .^{2}$ Environmental exposure to chemicals, including air pollutants, has been considered as one of the potential factors contributing to deteriorated semen quality, ${ }^{3}$ although the biological mechanisms remain uncertain. Many of the components of ambient particulate matter (PM), such as heavy metals and polycyclic aromatic hydrocarbons

\section{Key messages}

What is already known about this subject?

- The health effects of exposure to ambient fine particulate matter $\left(\mathrm{PM}_{2.5}\right)$ air pollution on semen quality are not clear.

What are the new findings?

- We investigated the health effects of exposure to fine $\mathrm{PM}_{2.5}$ on semen quality.

How might this impact on policy or clinical practice in the foreseeable future?

- Both short-term (a spermatogenic cycle, 3-month) and long-term (2-year) exposure to $\mathrm{PM}_{2.5}$ is associated with a lower level of sperm normal morphology and a higher level of sperm concentration.

- Exposure to ambient $\mathrm{PM}_{2.5}$ air pollution may serve as a risk factor of male reproductive health.

(PAHs), have been shown to have toxic effects on semen quality in animal experiments. ${ }^{4-6}$ Oxidative stress induced by particles is also of the potential to disturb spermatogenesis.

Air pollution has become the world's largest single environmental health risk. Exposure to ambient air pollution was estimated to cause $>3.7$ million premature deaths worldwide in 2012 . $^{7}$ PM is the most important air pollutant and affects more people than any other pollutants. There has been a large volume of epidemiological studies examining air pollution and its various health outcomes including birth defects. However, only a few studies with small samples have investigated the health effects of ambient PM on semen quality in humans, and the results have been inconsistent. ${ }^{8-15}$ In addition, most of these studies focused on the acute or short-term effects, despite the fact that people generally suffer from prolonged exposure. Chronic, low-dose exposure may contribute to significant spermatogenesis impairment. ${ }^{16}$ Moreover, previous studies have generally estimated PM exposure using residential proximity to routine ground-level air pollution monitoring stations. The exposure is typically at the community (district, county or city) rather than the individual level, with the same exposure level assigned to all of a community's. This limitation may mask the variation, or 
cause misclassification, thus leading to the inconsistent results of possible effects associated with PM air pollution. We therefore investigated the health effects of exposure (including long-term exposure and a spermatogenic cycle exposure) to fine PM- PM with an aerodynamic diameter of $<2.5 \mu \mathrm{m}\left(\mathrm{PM}_{2.5}\right)$-on semen quality in Taiwanese men from the general population, using a spatiotemporal model based on satellite-derived aerosol optical depth (AOD) data to estimate each participant's $\mathrm{PM}_{2.5}$ exposure.

\section{METHODS}

\section{Study population}

We studied male participants from a large cohort consisting of Taiwanese residents who participated in a standard medical examination programme run by a private firm (MJ Health Management Institution, Taipei, Taiwan). The details have been documented elsewhere. ${ }^{17-19}$ All procedures of the medical examination programme are approved according to ISO9001. Information on the medical examinations and related quality control can be accessed in the Technical Report of the MJ Health Research Foundation. ${ }^{20}$ In brief, the participants received a series of medical examinations including general physical examinations, anthropometric measurements and biochemical tests of blood and urine. They also completed a standard self-administered questionnaire survey. The semen quality assessment was voluntarily selected by the male participants. All of the participants gave written informed consent when they joined the programme.

There were 158542 male participants of reproductive age (15-49 years) $)^{21}$ who participated in the programme from 2001 to 2014, when satellite AOD data from the US National Aeronautics and Space Administration were available for air pollution exposure estimation. During the study period, 6938 participants voluntarily selected a semen quality examination. Generally, participants receiving semen measurements were comparable with all the 158542 male participants in terms of age, educational level, smoking habits and body mass index (BMI) (see online supplementary table 1). Only 211 participants $(3.0 \%)$ had multiple visits with two or more semen tests, therefore, repeated-measures analysis was not conducted. A total of 463 participants with incomplete information were excluded (1 on anthropometric measures, 3 on blood test results, 174 on educational level, 236 on lifestyle factors and 49 on $\mathrm{PM}_{2.5}$ exposure estimates due to missing address) and 6475 participants were ultimately included in data analysis. The 463 excluded participants had similar age and semen parameters (all $\mathrm{p}>0.05$ ).

\section{Semen analysis and health examination}

All of the participants were required to be sexually abstinent 2-7 days before contributing semen samples. They were then instructed to collect semen samples in sterile containers by masturbation. If the sample was collected at home, the participant was required to mark down the time and send the sample to laboratory immediately. No assessments were conducted if the duration was longer than 2 hours after ejaculation. The semen samples were analysed at the MJ Health Screening Center's central laboratory using standard protocols based on the WHO guidelines. ${ }^{21}$ Sperm concentration, total motility (percentage of motile sperm), progressive motility (percentage of sperm with progressive motility) and morphology (percentage of sperm of normal morphology) were assessed by trained technicians. The assessment began as soon as the ejaculates had liquefied, and were completed within 1 hour of sample collection. An aliquot of the sample was put into a diluent solution and thoroughly mixed for the concentration assessment. A microcell counting chamber was used in six areas at a total microscope magnification of $\times 400$. For the motility assessment, a $10 \mu \mathrm{L}$ well-mixed sample from another aliquot was placed on a glass slide covered with a coverslip, and then examined at a total microscope magnification of $\times 400$. Two hundred sperm cells were examined and classified into WHO motility categories A, B, C or D. The percentages of total motile sperm $(A+B+C)$ and sperm with progressive motility $(A+B)$ were calculated. Other smears were also prepared and air-dried, and then used for the morphology assessment. The WHO 'strict' criteria were used to examine 200 sperm cells and the percentage of morphologically normal sperm was calculated.

Details of health examination for other variables were described in previous publications. ${ }^{17-19}$

\section{Ambient $\mathrm{PM}_{2.5}$ estimate}

A spatiotemporal model with high resolution $(1 \times 1 \mathrm{~km})$ was developed based on satellite AOD data to retrieve ground-level $\mathrm{PM}_{2.5}$ concentrations. The details have been described elsewhere. ${ }^{17}$ This model was validated with ground-measured data from >70 monitoring stations in Taiwan from 2005 to 2014. $\left(\mathrm{PM}_{2.5}\right.$ data were only available for three monitoring stations from 2000 to 2004. Thus, validations were not conducted for this period.) The correlation coefficients ranged from 0.72 to 0.83 for yearly average $\mathrm{PM}_{2.5}$, and 0.70 to 0.81 for monthly average $\mathrm{PM}_{2.5}$ in different years (see online supplementary figure 1).

Each participant's address was geo-coded into latitude and longitude, and address-specific monthly and yearly average $\mathrm{PM}_{2.5}$ concentrations were calculated afterwards. The estimated annual average concentrations in Taiwan and at the participants' locations are presented in figure 1 . The southwestern and middle-eastern areas were generally the most and least heavily polluted, respectively. Most of the participants lived in the western area with apparent gradients of exposure. Because a spermatogenic cycle is around 3 months, we calculated 3 -month average concentration (based on the
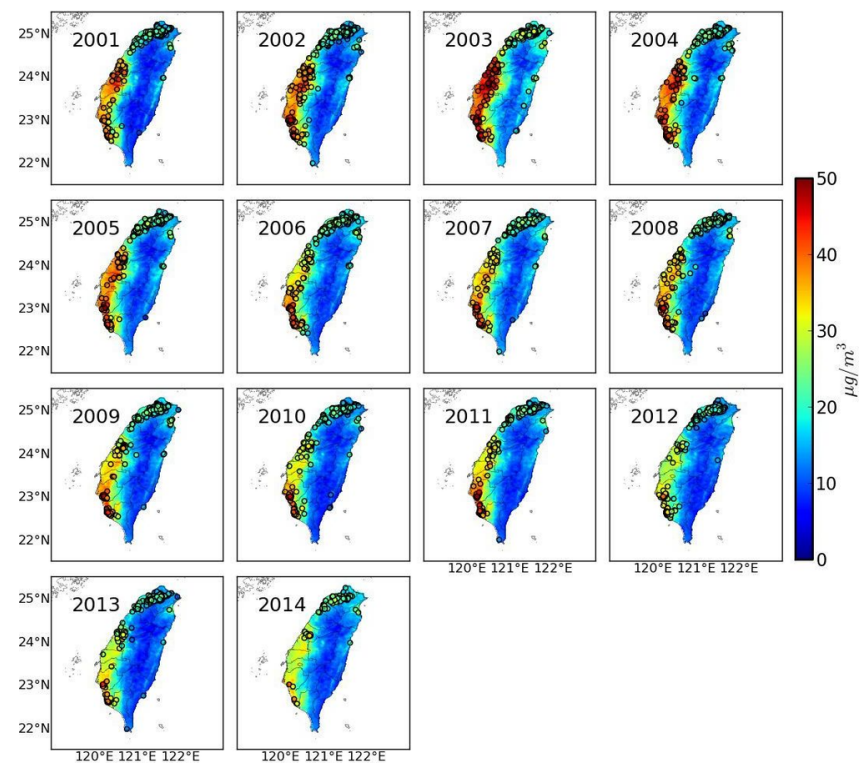

Figure 1 Map of the annual average concentration of satellite-based estimated $\mathrm{PM}_{2.5}$ in Taiwan between 2001 and 2014. Circles represent the locations of the study participants. PM $_{2.5}$, particulate matter with an aerodynamic diameter $<2.5 \mu \mathrm{m}$. 
concentrations of the month of the medical examination and the 2 months before the medical examination) to assess the short-term effects. We also calculated 2-year average concentration (based on the concentrations of the year of the medical examination and the year before the medical examination) as an indicator of long-term exposure to ambient $\mathrm{PM}_{2.5}$ air pollution.

\section{Statistical analysis}

We used multivariable linear regression models to evaluate the associations between $\mathrm{PM}_{2.5}$ exposure and each semen quality parameter. Sperm concentration was log-transformed for data analysis due to its skewed distribution. A crude model without adjustment and three adjusted models were developed with potential confounders added gradually: model 1 was adjusted for age (years), education (high school or lower, college or university and postgraduate), smoking status (never, former and current), alcohol consumption (less than once/week, 1-3 times/week and >3 times/week), exercise ( $<1$ hour/week, 1-2 hours/week and $>2$ hours/week) and occupational exposure to dust and organic solvents (yes and no); model 2 was further adjusted for BMI (calculated as weight $(\mathrm{kg})$ divided by the square of height $(\mathrm{m})$ ), systolic blood pressure, fasting plasma glucose and total cholesterol (all as continuous variables); model 3 was further adjusted for season (calendar season) and year of the medical examination (categorical variable). Short-term and long-term effects of $\mathrm{PM}_{2.5}$ exposure were analysed separately for comparison. The effect estimates were calculated for an increment of every $5 \mu \mathrm{g} / \mathrm{m}^{3}$ in average $\mathrm{PM}_{2.5}$ concentrations. The potential effect modifications by age group ( $<35$ years vs $\geq 35$ years), smoking (never vs ever) and overweight (BMI $\geq 25.0$ vs $<25.0 \mathrm{~kg} / \mathrm{m}^{2}$ ) were examined by adding multiplicative interactions terms between $\mathrm{PM}_{2.5}$ and these factors. Each potential modifier was examined in separate models.

We also used logistic regression models to investigate the associations between $\mathrm{PM}_{2.5}$ exposure and the risk of decreased semen parameters. Participants with the bottom 10\% of the parameters were defined as having decreased semen parameters. ORs were calculated for every $5 \mu \mathrm{g} / \mathrm{m}^{3}$ increment in $\mathrm{PM}_{2.5}$ concentrations.

We further performed subgroup analyses stratified by normal and abnormal semen parameters according to reference values from the WHO guidelines. ${ }^{22}$ A total of 1524 participants were categorised into abnormal group because they had at least one of the four semen parameters lower than the following reference limits: $15 \times 10^{6} / \mathrm{mL}$ for sperm concentration, $40 \%$ for total motility, $32 \%$ for progressive motility and $4 \%$ for normal morphology. Consequently, the other 4951 participants were categorised into normal group.

All of the statistical analyses were performed using statistical package R V.3.2.3 (R Core Team, Vienna, Austria). A two-tailed $\mathrm{p}$ value of $<0.05$ was considered to be statistically significant.

\section{RESULTS}

The general characteristics of the 6475 participants are presented in table 1 . The average age was 31.9 years (SD: 4.3) and they were well educated. The majority never smoked (64.2\%) and had alcohol use less than once a week (83.1\%).

The semen characteristics by year are shown in table 2 . For all of the participants, the mean (SD) values for sperm concentration, total motility, progressive motility and normal morphology
Table 1 General characteristics of Taiwanese men

\begin{tabular}{|c|c|}
\hline \multicolumn{2}{|l|}{ Characteristics $(n=6475)$} \\
\hline Age (year) & $31.9(4.3)$ \\
\hline \multicolumn{2}{|l|}{ Education } \\
\hline High school or lower & $791(12.2 \%)$ \\
\hline College or university & $4006(61.9 \%)$ \\
\hline Postgraduate & $1678(25.9 \%)$ \\
\hline \multicolumn{2}{|l|}{ Cigarette smoking } \\
\hline Never & $4158(64.2 \%)$ \\
\hline Former & $560(8.6 \%)$ \\
\hline Current & $1757(27.1 \%)$ \\
\hline \multicolumn{2}{|l|}{ Alcohol consumption } \\
\hline Less than once/week & $5383(83.1 \%)$ \\
\hline 1-3 times/week & $824(12.7 \%)$ \\
\hline$>3$ times/week & $268(4.1 \%)$ \\
\hline \multicolumn{2}{|l|}{ Exercise } \\
\hline$<1$ hour/week & $2780(42.9 \%)$ \\
\hline 1-2 hours/week & $2551(39.4 \%)$ \\
\hline$>2$ hours/week & $1144(17.7 \%)$ \\
\hline \multicolumn{2}{|l|}{ Occupational exposure } \\
\hline Dust & $386(6.0 \%)$ \\
\hline Organic solvent & $583(9.0 \%)$ \\
\hline Body mass index $\left(\mathrm{kg} / \mathrm{m}^{2}\right)$ & $23.9(3.3)$ \\
\hline Systolic blood pressure $(\mathrm{mm} \mathrm{Hg})$ & $118.6(13.1)$ \\
\hline Glucose (mg/dL) & $97.8(13.7)$ \\
\hline Total cholesterol (mg/dL) & $189.9(34.2)$ \\
\hline
\end{tabular}

Data are presented as the mean (SD) for continuous variables and the number (percentage) for categorical variables.

were $54.0(41.3) \times 10^{6} / \mathrm{mL}, 65.3(14.5) \%, 46.8(16.1) \%$ and $67.4(14.9) \%$, respectively. Linear regression models were used to investigate the trends in semen parameters over the study period adjusting for age. An increasing trend was observed for progressive motility $(\mathrm{p}<0.001)$ and no trends were found for other three parameters (all $\mathrm{p}>0.05$ ).

$\mathrm{PM}_{2.5}$ distribution among the participants is shown by year in figure 2 and locations of the study participants are shown in figure 1 . The $\mathrm{PM}_{2.5}$ concentrations varied spatially among the participants within each year. The overall mean 3-month and 2-year $\mathrm{PM}_{2.5}$ concentrations were 25.8 (SD: 8.5) and 26.1 (SD: 7.3$) \mu \mathrm{g} / \mathrm{m}^{3}$, with IQRs of 7.9 and $5.9 \mu \mathrm{g} / \mathrm{m}^{3}$, respectively. The 3-month and the 2-year $\mathrm{PM}_{2.5}$ concentration were highly correlated (correlation coefficient $=0.81, \mathrm{p}<0.001$ ).

The results of the regression models are summarised in table 3. Both short-term and long-term exposure had similar effects on sperm concentration and morphology. An increment of $5 \mu \mathrm{g} / \mathrm{m}^{3}$ in $\mathrm{PM}_{2.5}$ was associated with an increase in sperm concentration $\left(1.02 \times 10^{6} / \mathrm{mL}\right.$ for short-term exposure and $1.03 \times 10^{6} / \mathrm{mL}$ for long-term exposure) and reduced risk of decreased concentration (OR was 0.90 for both shortterm and long-term exposure). In contrast, an increment of $5 \mu \mathrm{g} / \mathrm{m}^{3}$ in $\mathrm{PM}_{2.5}$ was associated with a decrease in normal morphology $(0.83 \%$ for short-term exposure and $1.29 \%$ for long-term exposure) and increased risk of decreased normal morphology (ORs were 1.18 for short-term exposure and 1.26 for long-term exposure). There were no associations observed for progressive motility. Regarding the total motility, the association was not significant for short-term exposure in logistic regression analysis.

No significant effect modifications were observed for age group, smoking or overweight among the participants in relation 
Table 2 Distribution of semen parameters in Taiwanese men

\begin{tabular}{lclllll}
\hline Year & Participants & Age (year) & Concentration $\left(10^{6} / \mathbf{m L}\right)^{*}$ & Total motility (\%) & Progressive motility (\%) & Normal morphology (\%) \\
\hline 2001 & 501 & $30.8(4.2)$ & $41.9(2.3)$ & $66.4(13.7)$ & $46.9(15.3)$ & $71.8(13.7)$ \\
\hline 2002 & 611 & $31.1(4.5)$ & $41.8(2.3)$ & $65.5(12.9)$ & $45.7(15.2)$ & $66.5(16.0)$ \\
\hline 2003 & 558 & $31.5(4.3)$ & $42.5(2.4)$ & $64.8(14.3)$ & $46.4(15.6)$ & $67.3(16.7)$ \\
\hline 2004 & 535 & $31.1(4.4)$ & $40.6(2.5)$ & $65.3(14.0)$ & $48.4(15.4)$ & $67.9(15.2)$ \\
\hline 2005 & 519 & $31.5(3.9)$ & $38.3(2.4)$ & $65.6(14.2)$ & $46.5(15.4)$ & $70.7(13.5)$ \\
2006 & 530 & $31.3(4.0)$ & $39.1(2.2)$ & $64.8(13.6)$ & $45.7(15.4)$ & $65.7(13.4)$ \\
2007 & 517 & $32.0(3.8)$ & $38.6(2.6)$ & $66.8(12.7)$ & $46.5(15.1)$ & $65.8(13.5)$ \\
2008 & 556 & $32.3(4.2)$ & $38.6(2.4)$ & $64.8(14.9)$ & $45.9(15.9)$ & $65.8(14.4)$ \\
2009 & 437 & $32.5(4.4)$ & $39.3(2.5)$ & $64.5(14.8)$ & $43.9(14.4)$ & $64.0(14.3)$ \\
2010 & 447 & $32.7(4.0)$ & $39.8(2.5)$ & $64.2(14.5)$ & $44.4(15.6)$ & $63.7(14.3)$ \\
2011 & 424 & $32.8(4.2)$ & $40.3(2.3)$ & $65.8(15.4)$ & $49.1(18.0)$ & $66.3(14.9)$ \\
\hline 2012 & 402 & $33.0(4.5)$ & $44.3(2.3)$ & $64.2(16.5)$ & $48.1(17.6)$ & $67.8(16.4)$ \\
2013 & 322 & $33.2(4.1)$ & $44.1(2.2)$ & $66.1(16.6)$ & $53.4(19.7)$ & $72.1(13.1)$ \\
\hline 2014 & 116 & $32.7(4.4)$ & $45.9(2.1)$ & $64.1(19.5)$ & $47.1(17.0)$ & $77.0(12.4)$ \\
\hline All & 6475 & $31.9(4.3)$ & $40.6(2.4)$ & $65.3(14.5)$ & $46.8(16.1)$ & $67.4(14.9)$ \\
\hline$p$ for trend & - & - & 0.27 & 0.37 & $<0.001$ & 0.95 \\
\hline
\end{tabular}

Results are presented as the mean (SD) for the age and semen parameters.

*Sperm concentration was log-transformed to achieve normality for data analysis and then geometric mean (SD) were transformed back for presentation.

to the association between 3-month or 2-year $\mathrm{PM}_{2.5}$ and semen parameters (all $\mathrm{p}>0.10$ ).

The subgroup analyses generally yielded similar results (see online supplementary table 2 for participants with normal semen parameters and online supplementary table 3 for participants with abnormal semen parameters).

\section{DISCUSSION}

To our knowledge, this is the largest study investigating the health effects of $\mathrm{PM}_{2.5}$ air pollution on semen quality. It is also the first study considering the effects of both long-term exposure and short-term exposure (in a spermatogenic cycle). We

\section{3-month PM2.5 2-year PM2.5}

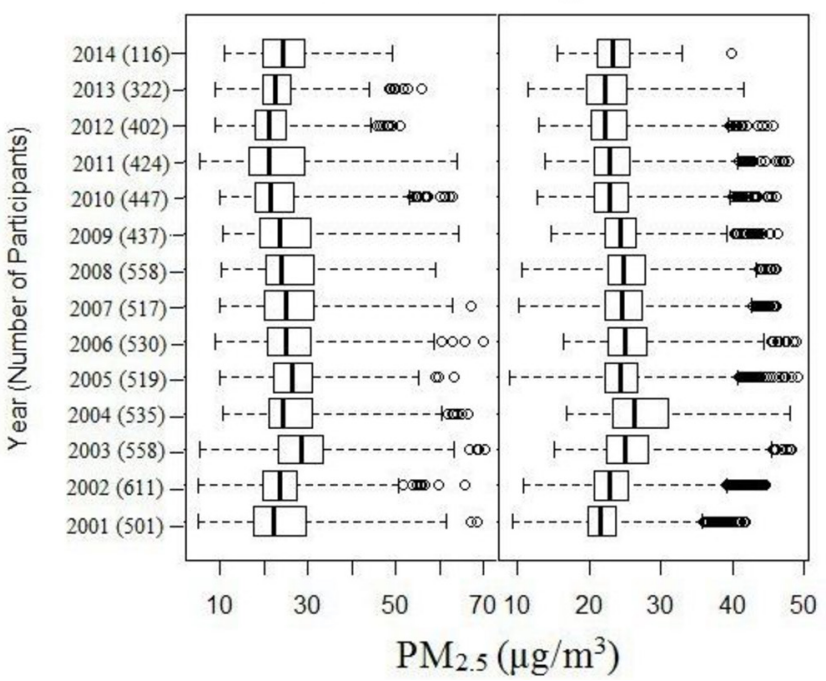

Figure 2 Distribution of $\mathrm{PM}_{2.5}$ from 2001 to 2014; 3-month refers to the month of and the 2 months before the visit; 2 -year refers to the year of and the year before the visit. Boxes cover the $25-75$ th percentile (IQR) with a centre line for the median concentration. Whiskers extend to the highest observation within 3 IQR of the box, with more extreme observations shown as circles. $\mathrm{PM}_{2.5^{\prime}}$ particulate matter with an aerodynamic diameter $<2.5 \mu \mathrm{m}$. find it is highly correlated between long-term and short-term exposure in this population. Because air pollution is quite stable over the study period, we speculate the exposure in a spermatogenic cycle is similar to the long-term exposure, and the effects are more likely the reflections of long-term exposure. We find robust associations between $\mathrm{PM}_{2.5}$ exposure and semen quality. Every increment of $5 \mu \mathrm{g} / \mathrm{m}^{3}$ in 2-year average $\mathrm{PM}_{2.5}$ is associated with a decrease of $1.29 \%$ in sperm normal morphology and a $26 \%$ increased risk of having the bottom $10 \%$ of sperm normal morphology, after adjusting for a wide range of potential confounders. In contrast, every increment of $5 \mu \mathrm{g} / \mathrm{m}^{3}$ in 2-year average $\mathrm{PM}_{2.5}$ is associated with an increase of $1.03 \times 10^{6} / \mathrm{mL}$ in sperm concentration and a $10 \%$ decreased risk of having the bottom $10 \%$ of sperm concentration. The associations remain robust in the analyses by taking into account a wide range of potential confounders, using different statistical methods (linear and logistic regression) and categorising participants into normal and abnormal groups.

The negative association between air pollution and normal morphology was also reported in the Teplice Program in Czech Republic, ${ }^{11}$ and in two recent studies in China and Poland. ${ }^{1013}$ The average time course in these studies was 3-month concentration. However, long-term exposure was not taken into account. It is not clear it was a short-term exposure or a surrogate of long-term exposure. Three studies in the USA, which the average time ranged from daily to 3 -month concentration, did not observe significant associations. ${ }^{8912}$ Hansen et al attributed the non-association to the relatively low level of air pollution. ${ }^{9}$

The mechanism of how air pollution adversely affects sperm morphology is not fully understood. Air pollution-induced oxidative stress has been hypothesised to be one of the contributors to sperm dysfunction. A wide range of air pollutants, such as $\mathrm{O}_{3}$, $\mathrm{NO}_{2}$ and particles, can induce increased oxidative stress, ${ }^{23}$ which is related to the excessive production of reactive oxygen species (ROS). ROS and their metabolites can damage DNA, lipids and proteins, altering enzymatic systems and cell apoptosis and, ultimately, lead to decreasing semen quality. ${ }^{24}$ In addition, $\mathrm{PM}_{2.5}$ may carry multiple trace elements and PAHs. The toxic effects of metals such as lead and cadmium on spermatogenesis have been well documented in animal models ${ }^{56}$ and confirmed in human 


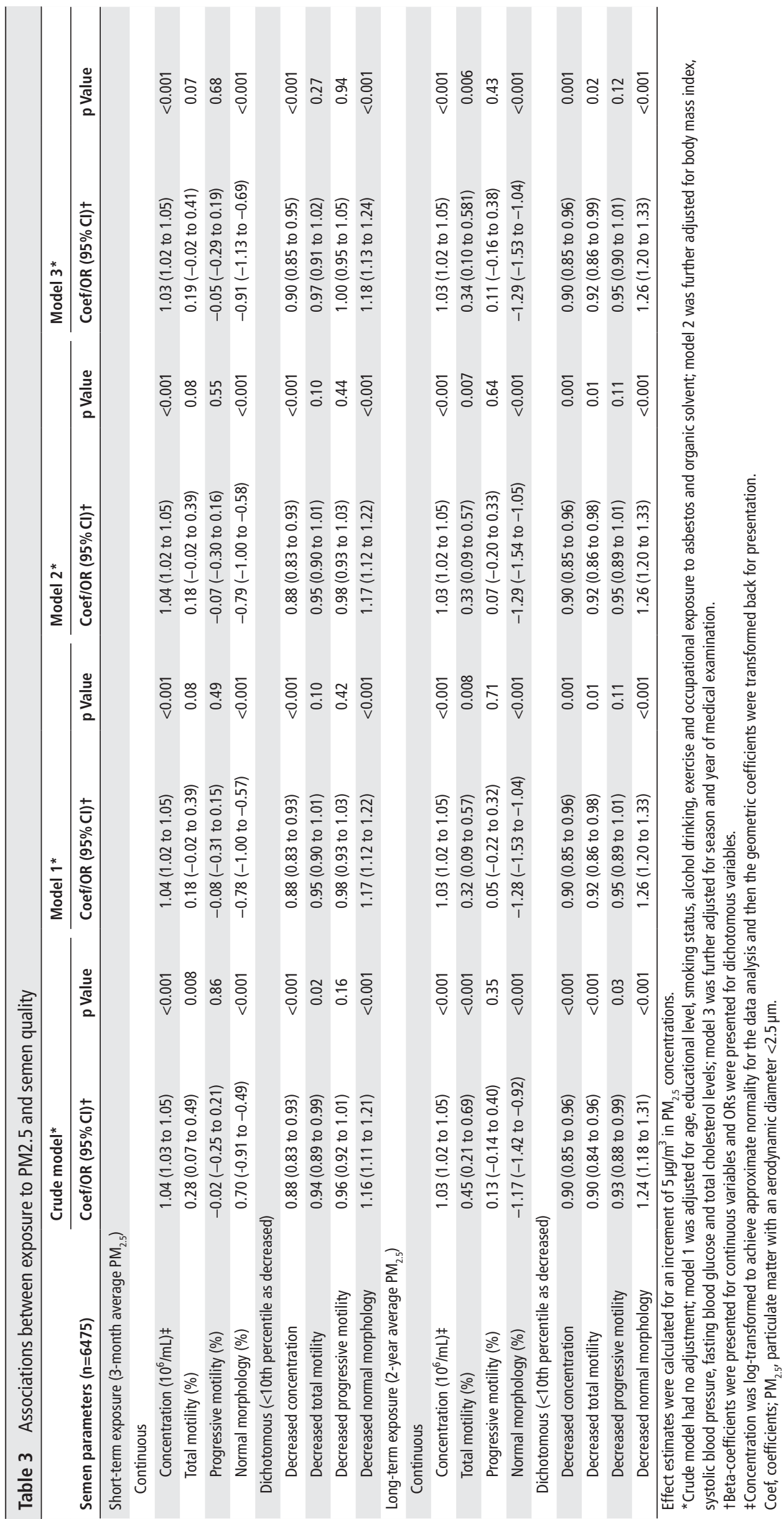


studies. ${ }^{25}$ PAHs, a group of compounds that includes several endocrine disruptors, can also cause sperm alterations through its effects on the hypothalamic pituitary axis and testicular spermatogenesis. ${ }^{4}$

We observed a positive association between exposure and sperm concentration. This finding is not in line with previous studies, most of which have not observed significant associations between air pollution and sperm concentration. ${ }^{8911}$ Sokol et al reported a negative association between $\mathrm{O}_{3}$ and sperm concentration. ${ }^{12} \mathrm{~A}$ recent study by Zhou et al reported a positive association between sperm concentration and $\mathrm{PM}_{10}{ }^{13}$ which is similar to our study. Regarding motility, the associations also remain uncertain. Hammoud et al and Selevan et al observed a negative association, ${ }^{811}$ whereas no associations were observed in other studies. ${ }^{1012} 13$ The reasons for the positive associations between exposure and sperm concentration in the present study need to be elucidated by further studies. We hypothesise that the slight increases in sperm concentration are a compensatory phenomenon. Numerous studies have demonstrated the hormesis phenomenon on chemical aerosol exposure and follicle-stimulating hormone (FSH) and luteinising hormone (LH) ${ }^{26}$ Low-dose exposure may increase the levels of FSH and LH, which strengthen spermatogenesis, thus increasing sperm concentration. Further studies are warranted to investigate the underlying mechanism.

We did not observe decline trends in semen quality over the study period. Although the trend of progressive motility was statistically significant, the figures fluctuated over time. The reported global downwards trend in semen quality has not been concluded because previous studies exhibit great heterogeneity due to geographical and/or ethnic variations and different study designs and methodological standards. ${ }^{27}$ In the present study, we adjusted for the year in the data analyses and the adjustment had few effects.

Compared with previous studies, our study has some important strengths. The large sample size enabled us to have sufficient power to detect the small effects by low-dose exposure. Furthermore, our participants were from a general population rather than being men from infertility clinics. The affluent information on a wide range of potential confounders and modifiers enabled us to consider the effects of these factors. Another advantage is that we used accurate, high-resolution satellite-based technology to estimate $\mathrm{PM}_{25}$ exposure at the individual level. All of the previous studies estimated air pollution exposure at the community level, which may mask exposure variation and result in misclassification. This technology also enables us to obtain short-term and long-term exposure for comparison.

However, there are limitations. First, all of the participants in this study were volunteers, and information on their history of infertility diseases was not available. We therefore could not exclude the possible influence of infertility disorders for some of the participants, but it should not affect our conclusions because $\mathrm{PM}_{2.5}$ exposure was unlikely to be differentially distributed among fertile and infertile participants. In addition, we got similar results in subgroup analyses stratified by normal and abnormal semen parameters. Given that the participants were well educated, we should be cautious when generalising the findings into general populations. Second, most participants (97\%) had only one semen measurement and this limited us to perform a repeated-measures analysis, which would address within-person variations over time. Another limitation is that we only estimated ambient $\mathrm{PM}_{2.5}$ levels. Information on indoor PM ${ }_{25}$ was not available. Despite evidence that indoor and outdoor PM levels are highly correlated, ${ }^{28}{ }^{29}$ we cannot exclude the possible influence of indoor PM sources on some of the participants. In addition, the $\mathrm{PM}_{2.5}$ concentrations were calculated at the fixed addresses and participants' activity pattern was not taken into account. More advanced techniques technologies are needed for more accurate exposure assessment. Our study also lacked data on other gaseous air pollutants and future studies need to take it into consideration. Finally, the longterm and short-term (a spermatogenic cycle) exposure are highly correlated in the present study. We are not able to distinguish the effects of short-term exposure.

\section{CONCLUSIONS}

In conclusion, we found a robust association between exposure to $\mathrm{PM}_{2.5}$ air pollution and low percentage of sperm normal morphology in reproductive-age men. Although the effect estimates are small and the significance might be negligible in a clinical setting, this is an important public health challenge. Given the ubiquity of exposure to air pollution, a small effect size of $\mathrm{PM}_{2.5}$ on sperm normal morphology may result in a significant number of couples with infertility. We advocate global strategies on mitigation of air pollution to improve reproductive health. In contradiction to the negative associations with morphology, we observed exposure to $\mathrm{PM}_{2.5}$ is associated with a higher level of sperm concentrations. We speculate the positive associations may be due to the compensation mechanism. Further studies are warranted to investigate this phenomenon and its underlying mechanism.

\section{Author affiliations}

${ }^{1} J o c k e y$ Club School of Public Health and Primary Care, The Chinese University of Hong Kong, Hong Kong, China

${ }^{2}$ Shenzhen Research Institute of the Chinese University of Hong Kong, Shenzhen, China

${ }^{3}$ Division of Environment, The Hong Kong University of Science and Technology, Hong Kong, China

${ }^{4}$ Department of Civil and Environmental Engineering, The Hong Kong University of Science and Technology, Hong Kong, China

${ }^{5}$ Research Center for Humanities and Social Sciences, Academia Sinica, Taiwan

${ }^{6}$ MJ Health Research Foundation, MJ Group, Taipei, Taiwan

${ }^{7}$ Department of Sociology, The Chinese University of Hong Kong, Hong Kong, China

${ }^{8}$ Institute for Risk Assessment Sciences, Utrecht University, The Netherlands

${ }^{9}$ School of Public Health, Fudan University, Shanghai, China

${ }^{10}$ Institute of Sociology, Academia Sinica, Taiwan

Acknowledgements The authors would like to thank MJ Health Research Foundation for the authorisation of using MJ Health data (authorisation code: MJHR2015002A). The authors are grateful to the anonymous reviewers and the editor for their valuable comments.

Contributors XQL conceived the study. LC, AKHL and XQL designed the research and acquired the data. ZZ conducted statistical analysis. ZZ and XQL drafted the manuscript. All authors made critical revision of the manuscript.

Funding This work was partially supported by Environmental Health Research Fund of the Chinese University of Hong Kong (7104946).

Disclaimer Any interpretation or conclusion related to this manuscript does not represent the views of MJ Health Research Foundation.

\section{Competing interests None declared.}

Ethics approval The Joint Chinese University of Hong Kong — New Territories East Cluster Clinical Research Ethics Committee.

Provenance and peer review Not commissioned; externally peer reviewed.

Open Access This is an Open Access article distributed in accordance with the Creative Commons Attribution Non Commercial (CC BY-NC 4.0) license, which permits others to distribute, remix, adapt, build upon this work non-commercially, and license their derivative works on different terms, provided the original work is properly cited and the use is non-commercial. See: http://creativecommons.org/ licenses/by-nc/4.0/

(c) Article author(s) (or their employer(s) unless otherwise stated in the text of the article) 2018. All rights reserved. No commercial use is permitted unless otherwise expressly granted.

\section{REFERENCES}

1 Mascarenhas MN, Flaxman SR, Boerma T, et al. National, regional, and global trends in infertility prevalence since 1990: a systematic analysis of 277 health surveys. PLoS Med 2012;9:e1001356. 
2 World Health Organization. WHO laboratory manual for the examination and processing of human semen. 5th edn. Switzerland:WHO Press, 2010.

3 Jurewicz J, Hanke W, Radwan M, et al. Environmental factors and semen quality. Int J Occup Med Environ Health 2009;22:305-29.

4 Jeng HA, Yu L. Alteration of sperm quality and hormone levels by polycyclic aromatic hydrocarbons on airborne particulate particles. J Environ Sci Health A Tox Hazard Subst Environ Eng 2008;43:675-81.

5 Rafique M, Khan N, Perveen K, et al. The effects of lead and zinc on the quality of semen of albino rats. J Coll Physicians Surg Pak 2009;19:510-3.

6 Roychoudhury S, Massanyi P, Bulla J, et al. Cadmium toxicity at low concentration on rabbit spermatozoa motility, morphology and membrane integrity in vitro. J Environ Sci Health A Tox Hazard Subst Environ Eng 2010;45:1374-83.

7 World Health Organization. Ambient (outdoor) air quality and health. http://www. who.int/mediacentre/factsheets/fs313/en/ (assessed 20 feb 2017).

8 Hammoud A, Carrell DT, Gibson M, et al. Decreased sperm motility is associated with air pollution in Salt Lake City. Fertil Steril 2010;93:1875-9.

9 Hansen C, Luben TJ, Sacks JD, et al. The effect of ambient air pollution on sperm quality. Environ Health Perspect 2010;118:203-9.

10 Radwan M, Jurewicz J, Polańska K, et al. Exposure to ambient air pollution--does it affect semen quality and the level of reproductive hormones? Ann Hum Biol 2016:43:50-6.

11 Selevan SG, Borkovec L, Slott VL, et al. Semen quality and reproductive health of young Czech men exposed to seasonal air pollution. Environ Health Perspect 2000;108:887-94.

12 Sokol RZ, Kraft P, Fowler IM, et al. Exposure to environmental ozone alters semen quality. Environ Health Perspect 2006;114:360-5.

13 Zhou N, Cui Z, Yang S, et al. Air pollution and decreased semen quality: a comparative study of Chongqing urban and rural areas. Environ Pollut 2014;187:145-52.

14 Deng Z, Chen F, Zhang M, et al. Association between air pollution and sperm quality: A systematic review and meta-analysis. Environ Pollut 2016;208:663-9.

15 Lafuente R, García-Blàquez N, Jacquemin B, et al. Outdoor air pollution and sperm quality. Fertil Steril 2016;106:880-96.

16 Gabrielsen JS, Tanrikut C. Chronic exposures and male fertility: the impacts of environment, diet, and drug use on spermatogenesis. Andrology 2016;4:648-61.
17 Wen CP, Cheng TY, Tsai MK, et al. All-cause mortality attributable to chronic kidney disease: a prospective cohort study based on 462293 adults in Taiwan. Lancet 2008;371:2173-82.

18 Wu X, Tsai SP, Tsao CK, et al. Cohort Profile: The Taiwan MJ Cohort: half a million Chinese with repeated health surveillance data. Int J Epidemiol 2017.

19 Zhang Z, Chang L-yun, Lau AKH, et al. Satellite-based estimates of long-term exposure to fine particulate matter are associated with C-reactive protein in 30034 Taiwanese adults. Int J Epidemio/ 2017;46:1126-36.

20 Chang L, Tsai SP, Wang ML, et al. MJ Health Database, MJ Health Research Foundation Technical Report, MJHRF-TR-01. 2017. http://www.mjhrf.org/file/en/ report/MJHRF-TR-01MJ\%20Health\%20Database.pdf (assessed 28 feb 2017).

21 World Health Organization. Manual for the Examination of Human Semen and Semencervical Mucus Interaction. 4th edition. New York: NY, USA: Cambridge University Press, 1999.

22 Cooper TG, Noonan E, von Eckardstein S, et al. World Health Organization reference values for human semen characteristics. Hum Reprod Update 2010;16:231-45.

23 Kelly FJ. Oxidative stress: its role in air pollution and adverse health effects. Occup Environ Med 2003;60:612-6.

24 Agarwal A, Virk G, Ong C, et al. Effect of oxidative stress on male reproduction. World J Mens Health 2014;32:1-17.

25 Pant N, Kumar G, Upadhyay AD, et al. Reproductive toxicity of lead, cadmium, and phthalate exposure in men. Environ Sci Pollut Res Int 2014;21:11066-74.

26 Tomei G, Tomao E, Ciarrocca M, et al. Follicle-stimulating hormone levels in male workers exposed to urban chemical, physical, and psychosocial stressors. Toxicol Ind Health 2009;25:395-402.

27 Merzenich $\mathrm{H}$, Zeeb H, Blettner M. Decreasing sperm quality: a global problem? BMC Public Health 2010;10:24.

28 Gotschi T, Oglesby L, Mathys P, et al. Comparison of black smoke and PM2.5 levels in indoor and outdoor environments of four European cities. Environ Sci Technol 2002;36:1191-7.

29 Massey D, Masih J, Kulshrestha A, et al. Indoor/outdoor relationship of fine particles less than 2.5 $\mathrm{mm}$ (PM2.5) in residential homes locations in central Indian region. Build Environ 2009;44:2037-45. 